\title{
EVOLVABLE PRODUCTION SYSTEMS: APPROACH TOWARDS MODERN PRODUCTION SYSTEMS
}

\author{
Pedro Neves \\ New University of Lisbon \\ pedromsneves@gmail.com
}

\author{
Mauro Onori \\ Royal Institute of Technology \\ onori@iip.kth.se
}

\begin{abstract}
To face current socio-economic adverse conditions enterprises must increase their efficiency and evolve to the requirements of customization and sustainability. Modern production systems need to deal with instability of markets and resource scarcity regarding an economical, ecological and social concern. The Evolvable Production Systems (EPS) has aimed at developing such technological solutions and support mechanisms that may endow European assembly companies to fulfil these demands. EPS seeks highly adaptable control and mechanical systems enhancing re-usability and interoperability of modules, extending their life cycle and enabling short deployment times at shop floor level. Only with very agile and adaptable systems will be possible to enhance material reusability, reduce energy consumption and give a quick response to unforeseen changed conditions or new business opportunities. This paper will present Evolvable Production Systems advantages in order to achieve such results towards more ecological and economically suitable production systems.
\end{abstract}

\section{KEYWORDS}

Evolvable Assembly Systems, Economy, Ecology

\section{INTRODUCTION}

The world is evolving every day imposing new constraints and new demands in a blink of an eye. This imposes the emergence of two fundamental skills: Adaptability and Agility.

Recent manufacturing roadmaps such as Eupass, Manufuture and Futman stress current Production Systems limitations, emphasizing social, economical and ecologic impacts of such systems. Modern production systems need to deal with instability of markets and resource scarcity regarding an economical, ecological and social concern.

Hence, research must be oriented towards sustainable manufacturing by focusing on economic, ecologic and social effectiveness of business. In Manufuture roadmap is highlighted the need to shift from capital and resource intensive societies to knowledge and service intensive ones. The short term approach where a manufacturing system is built from scratch (or almost) for each new product must be re-placed since it enhances resources and knowledge waste which lead to inefficiency. Manufacturing systems must therefore be designed and developed considering a long term approach enhancing re-usability of components and reconfigurability of systems making use of services and modules already available and promoting the use of all the knowledge acquired from the previous systems. This knowledge is valuable for the upper organisational levels of the enterprise since it can help improving the effectiveness of the business in the long run. Hence, enterprises must pursuit manufacturing agility from management to shop floor level. The long term success of an enterprise depends on its ability to produce innovative products with good quality, competitive cost and adequate time frame. Hence enterprises seek for 
more efficient organizational dynamics what led to the emergence of several types of networked interactions: supply chains, extended enterprises, virtual enterprises, collaborative networks, collaborative automation, etc. Such interactions can help enterprises improving agility by forming alliances enabling them to gain competitive advantage over competitors and react quicker to new business opportunities. Current major roadmaps have all clearly underlined that true industrial sustainability must be pursuit. Organisational sustainability is "the ability of an organisation to design its systems so that they address various requirements of all stakeholders to enable organisations sustain competitive advantage and not lead (in future) to diminished quality of life due to depletion of natural resources, loss of future economic opportunities, and adverse impacts on social system due to its operations" (WCED, 1987). Achieving sustainability is a mean for an enterprise to maintain competitiveness since it regards the adequate use of resources (economical, social and environmental), decreasing social and environmental impact of business while ensuring economic benefits.

In line with these aspects, the Evolvable Assembly System paradigm is attempting to develop technological solutions (mechanical and control) and support mechanisms (Ontology, Methodologies, Reference Architecture, etc.) that may endow European assembly companies to fulfil these demands remaining competitive economically and in the forefront in ecological matters.

\section{EVOLVABLE PRODUCTION SYSTEMS}

Assembly companies' major problems are related to uncertainty. It's difficult for companies to predict the type and range of products that will have to be developed, volumes of production and lifespan of the products. For these reasons, in order to be competitive and survive, companies need to optimize the cost and the time to market. Customization and the shorter life time of products demand a continuous adaptation of the manufacturing systems.

These issues had been addressed in several approaches (Flexible Manufacturing Systems (FMS), Re-configurable Manufacturing Systems (RMS), Holonic Manufacturing Systems (HMS), etc.) with little success to date (Onori et al.)

FMS attained efficient results in predefined foreseen situations during the system design, however the control solutions were too rigid and couldn't cope with unpredictable scenarios(new features imposed by market demands) imposing a great programming effort and therefore increasing the installation time and cost (Maffei et al., 2009). RMS considered the need to improve interoperability and lower the costs promoting the use of modular systems. Notwithstanding with such progress the modularization in such systems was based on assembly functions (feeding, handling, etc.) which is not suitable for a dynamic control solution (Maffei et al., 2009). HMS started with a very biological inspired approach (similar to EPS), however implementations along the years got deviated it from the basic approach resulting in more hierarchical systems. Despite having some similarities HMS and EPS paradigms diverge in several points and only EPS can achieve fine granularity (Onori and Barata, 2009). According to Wagner and Altenberg, for adaptation to occur the systems must possess evolvability, which can be defined as "the genome's ability to produce adaptive variants when acted upon the genetic system" (Wagner and Altenberg, 1996) or "the ability of an organism to adapt through evolutionary changes to new or altered environments" (Bowers, 2005). It is also highlighted by Wagner and Altenberg (1996) that adaptation is possible if improvements can be made in a stepwise approach (through evolution), demanding that improvements in one part of the system should not compromise past achievements. Modularity can enhance evolution of systems and avoid that improvements in one part of the system jeopardize a proper evolution of the system as whole. This is supported by Bowers (2005), "modularity is simply a consequence of the fitness of an individual such that fitter individuals tend to result in increased modularity". This supports that modern production systems should seek adaptation through the use of a modular approach where the individual modules that compose the system possess evolvability and therefore give evolvability to the system. Hence production systems can evolve, following a "fitness function" in order to accommodate disturbances and meet performance requirements, i.e. to adapt to changed conditions. Evolvable Assembly System paradigm is based on this principle and being evolvability a system concept, is envisaged to address every aspect of an assembly system throughout its life cycle, i.e., design and development, operation and evolution.

EPS paradigm derives from a more dynamic and mutable approach focusing on the changes in the production systems and how they can be managed efficiently. However it's important to underline that that EPS is not a generic solution but it is rather a specific approach that may be adopted by several products of the same class. To aim at a real flexible system (system that can fully adapt to unforeseen changes in environment conditions) the sub-systems and the control systems must be extremely adaptable. The lower a component is positioned in 
the hierarchic structure of the system the more flexible it must be in order to empower the system's flexibility (Maffei et al., 2009). The following figure illustrates precisely this, showing the relation between the complexity level of the system and the needed level of adaptability for it, emphasizing the aspect that control requires a high level of adaptability.
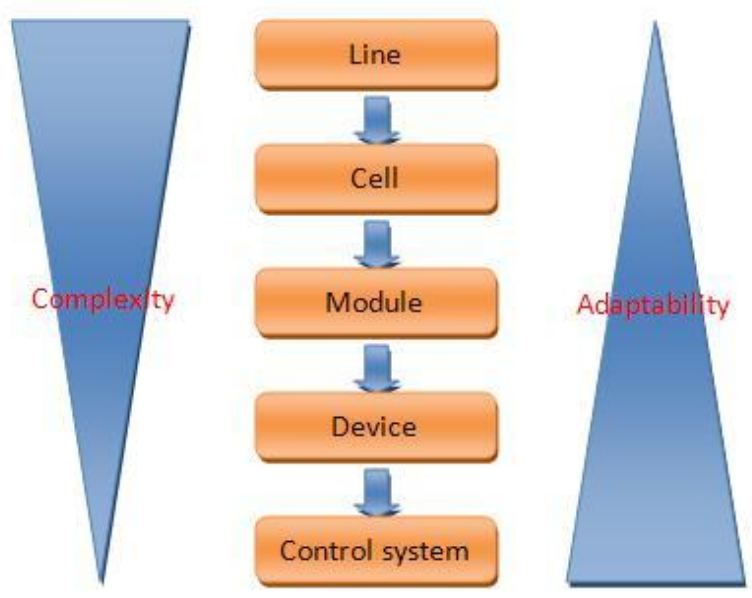

Figure 1 - Adaptability required at different system levels

The integration between the mechanical and control system is crucial since it's impossible to achieve an Adaptable Mechanical System if you don't have a very Adaptable Control System. In terms of system characteristics, there has been quite a wide range of discussions on Plug \& Produce, interoperability, reconfigurability, and other aspects. However, there is a gradual demand being posed on the system components that has to be accommodated, tested, and validated before one may claim to present any given characteristic. For example, as shown below, the Evolvable Assembly System work may, at this point in time, only truly claim to achieve some level of self-configurability (demands distributed control system and specialized interfaces). As one increases the demands on adaptability, the corresponding technology level must also be attained: selforganising systems demand, at the very least, that all components have embedded control that communicates through the distributed control approach. Likewise, if one claims self-diagnostics, the actual system must at least apply some form of autonomous module parameter feedback \& update. Therefore, full adaptability remains extremely linked to control and process issues (which is commonly not underlined).

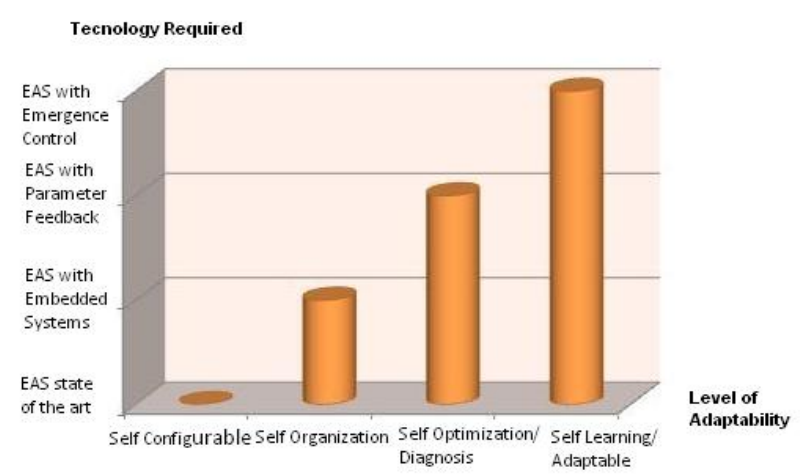

Figure 2 - Control System aimed by EAS

Figure 3 simply corroborates the previous figure at full System level: fault-tolerant Systems with reconfigurability must have components with embedded control, operating with distributed control, and with autonomous parameter feedback. Hence, true adaptability/flexibility still requires control R\&D.

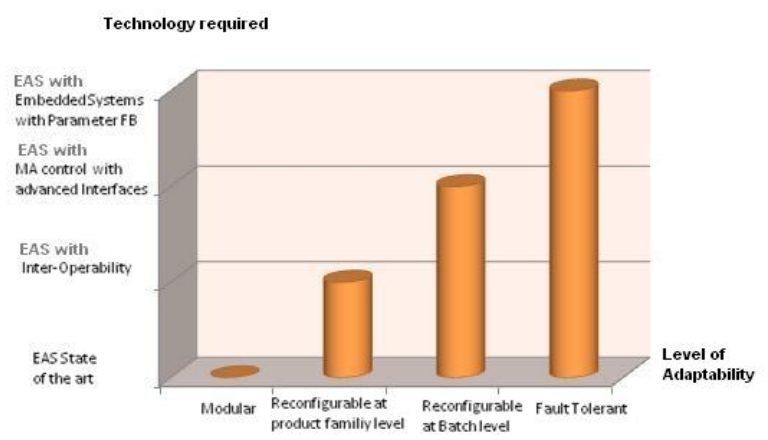

Figure 3 - Mechanical System aimed by EAS

EPS addresses these aspects aiming at systems that enhance continuous evolution and fast reconfigurability through the use of re-configurable, task-specific, process-oriented modules. EPS implements an agent based control where individual modules are endowed of several skills and are able to cooperate with other modules creating new skills enhancing interoperability and emergent behaviour.

Nevertheless EPS needs to guarantee agility and evolvability at management and business processes level in order to cope with the constant changes imposed by the market. Due to EPS processoriented modular approach it's possible to know à priori the skills available and which module provides the referred skill. This feature helps setting constraints to the product designer that must account them in order to maximize the efficiency of modules available enhancing re-usability of modules and reducing costs. The efficiency is maximized since a new module will only be developed if it is strictly necessary because similar results of the process cannot be achieved through the use of other modules 
or the process design of the product cannot be changed (Maffei et al., 2009). This is illustrated in the model below showing the process flow for system development when a new product idea emerges. The product design phase is enhanced by the use of general ontology (G.O.) and the System Modules (S.M.) that contains all the process oriented modules developed to date, and also by the interaction between the product designer and the system designer.

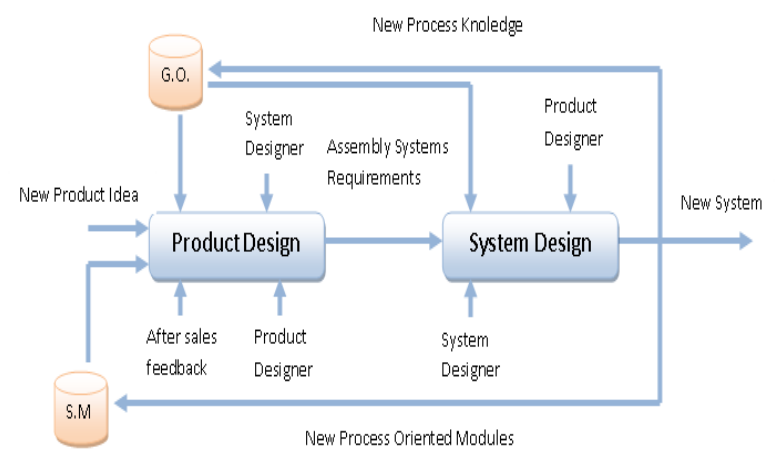

Figure 4 - Development in EPS approach

With this approach EPS strengthens the link between product and system design phases, establishing the modules as drivers for the product design which leads to several advantages:

1. Lower investment costs and risk factor by new modules development

2. Shorter deployment times and less risk in system installation and integration

3. Improvement of cost effectiveness

4. Ecology improvement

5. Increase of process knowledge since the assembly systems are better structured

In the next sections a deeper analysis will be granted on the advantages of EPS approach towards attaining more ecological and economic production systems.

\section{EVOLVABLE PRODUCTION SYSTEMS TOWARDS ECONOMICAL PRODUCTION SYSTEMS}

Achieving high adaptability to be able to cope with the constant changing market demands is a high priority of companies to remain competitive. High adaptability at factory level through the development of self-learning, self-optimizing and co-operative control systems is the response to turbulent and constant changing of tasks and products. When requirements of new products emerge there is a need for fast re-configurability on the assembly line in order to start the production fast and achieve low TTM (Time to Market) winning advantage over the competitors (Maffei et al., 2009). Besides shortening the TTM and winning advantage over competitors, the cost effectiveness can be highly improved using Evolvable Production Systems. Due to the modular and service-oriented approach of EPS there is a high re-usability of modules. This leads to costs reduction in human workforce (building new modules, re-configuring assembly line and programming new and old modules integrating the whole system), energy (energy consumption associated with the whole process), materials (materials needed to build new modules) and transport (transportation of materials or modules and associated costs of logistics). A study performed in the domestic appliances manufacturing sector has shown that up to $80 \%$ of the total manufacturing costs (apart from those of raw materials and off-the-shelf components) for production along an automated line do not come from the cost of the equipment installed on the line, but from costs related to both the initial system integration and/or to the integrated modification of the line as a result of changes in production, etc (Onori, 2009). Therefore EPS main advantage towards cost effectiveness lies in development of automated manufacturing lines that will greatly reduce, or even totally eliminate, the costs above mentioned, through a swift and adequate response to changing manufacturing conditions.

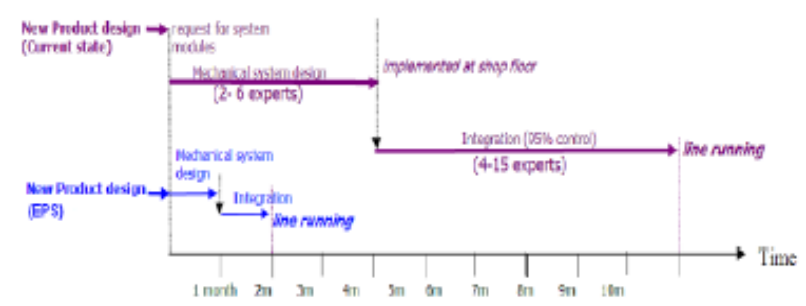

Figure 5 - Time scales in system deployment

To cope with actual dynamic markets and business, companies must be agile in the re-engineering process since it's likely that business opportunities arises fast, involving changes at shop floor level such as product change, changes at quality, new procedures, etc. EPS methodology is based on a holistic product/production lifecycle approach enabling efficient re-engineering practices. Strengthen the link between product and system design phases is one of the advantages of this approach because if the assembly process influence the way the product is designed then standardization and cost-reductions can be achieved (Onori et al., 2008).

Another aspect that EPS pursuits is the strength of the link between shop floor and management level of an enterprise. If this is achieved important data 
from the shop floor can be exported to higher organizational levels to support decision making and promote supply chain and enterprise agility. Hence with high level of adaptability at shop floor level and accurate business tools to support shop floor's integration with higher enterprise levels it can be targeted to quicker address new business opportunities and/or accommodate the required actions due to changes in the business/production environment.

\section{EVOLVABLE PRODUCTION SYSTEMS TOWARDS ECOLOGICAL PRODUCTION SYSTEMS}

The analysis of ecological impact is nowadays an important decision factor for companies operating production systems. There is a rising concern about oil and water scarcity and its consequences in industrial development. This problem is particularly addressed by the EUPASS roadmap underlining the fact that in the future conflicts might arise as consequence for impossibility to satisfy all the parties' needs. The water scarcity problem is an issue that must be accounted very seriously since industries in developing countries might find themselves in the situation where they have to compete with household and agricultural water users for the few water resources available. The figure below shows the water stress indicator provided by the Watergap2.0 report and this figure shows that several industrial regions are already in very high stress.

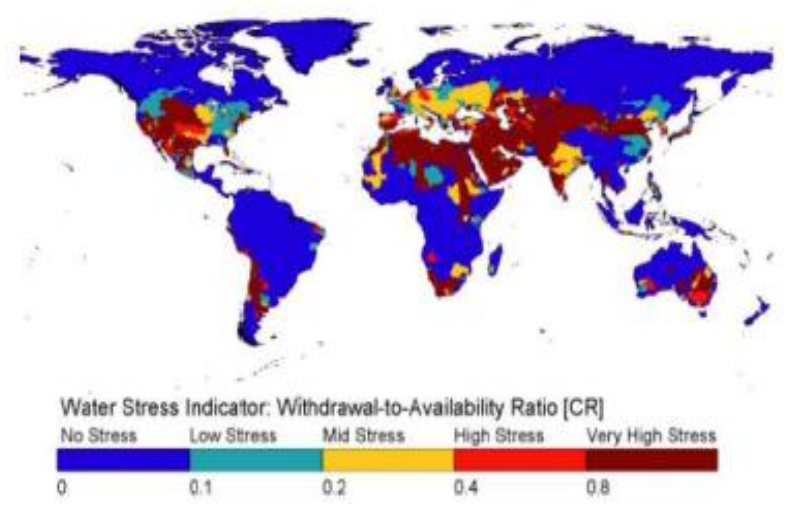

Figure 6 - Water stress indicator

Figure 7 was based on Energy Information Administration report of 2005 and it shows the history and projections of energy consumption. One can observe that the energy consumption have been rising along the years and the projection reflects that it will keep rising with time.

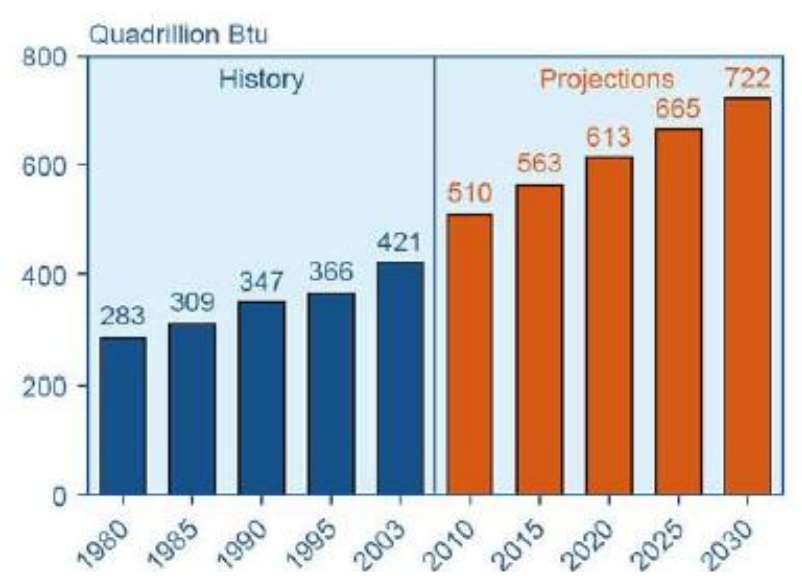

Figure 7 - Energy consumption history and projection

If production of modules is reduced by enhancing the re-usability of modules a significant amount of energy could be saved since it contributes significantly for global energy consumption. To struggle with scarcity of essential resources (being water and oil in the frontline) modern production systems must address adaptability and agility to cope with sustainability issues. Only with very agile and adaptable systems will be possible to enhance material re-usability and reduce energy consumption remaining economically competitive.

\section{CONCLUSION}

There is an urgent need for technologies and support mechanisms that may allow end-user to automate their operations at their own rate, with high reliability and minimal setup times. Only this way European SMEs can remain competitive and keep production in Europe avoiding more job cuts, helping tackle the actual financial crisis and enhance environment protection. At the same time an effort must be made towards moving to a more resourcesaving production since the ecological, economic, social and political consequences of oil and water scarcity can become unbearable and lead to conflicts. Latest manufacturing roadmaps such as EUPASS, Manufuture and Futman stress out these matters, underlining that enterprises should change systems development from reaction to short-term events to long term strategic development in order to be more ecological and economically efficient and achieve true sustainability. Hence, modern production systems must address adaptability and agility to cope with sustainability issues while regarding at instable markets and society demands. Only with very agile and adaptable systems will be possible to enhance material reusability, reduce energy consumption and give a 
quick response to unforeseen changed conditions or new business opportunities.

This paper presented the Economic and Ecologic impact of such solutions and the advantages of EPS approach towards a modern generation of production systems that can ease difficulties caused by turbulent markets, hard economic conjecture and ecologic demands such as water and oil scarcity.

\section{REFERENCES}

Barata J., Camarinha-Matos L., Onori M.; “A multi-agent based control approach for Evolvable Assembly Systems"; 3rd IEEE International Conference on Industrial Informatics (INDIN);2005

Barata J., Onori M., Frei R., Leitão P.; "Evolvable Production Systems:Enabling Research Domains";CARV 2007

Barata J, Ribeiro L, Onori M. ; "Diagnosis on Evolvable Production Systems"; Proc ISIE'07 - IEEE International Symposium on Industrial Electronics Vigo - Spain, 4-7 June, 2007

Bowers, C.P.; "Formation of Modules in a Computational Model of Embryogeny"; Proceedings of the 2005 Congress on Evolutionary Computation, Vol. 1, 2-5 September 2005, pp.537-542, IEEE Press

http://timeforchange.org/prediction-of-energyconsumption; checked on 10/5/2009

Maffei A., Dencker K., Bjelkemyr M., Onori M.; "From flexibility to Evolvability: ways to achieve selfreconfigurability and full-autonomy"; Syroco 2009

Maffei A., Onori M.; "A Preliminary Study of Business Model for Evolvable Production Systems"; (in press) 2009 IEEE/ISAM conference Seoul Korea

"Manufacturing Visions Policy Summary and Recommendations"; Manvis Report No. 6; November 2005

Onori M.; "Evolvable Assembly Systems - A New Paradigm?"; Proceedings of the 33rd International Symposium on Robotics (ISR2002); Stockholm, Sweden, Oct.2002

Onori M.; "Outlook Report on the Future of European Assembly Automation"; Project Report-Public, Document 1.5b, EUPASS-Evolvable Ultra Precision Assembly, NMP-2-CT-2004-507978; January 2009

Onori M, Maffei A, Barata J.; "Evolvable Assembly systems: latest developments"; The 8th International Conference on Evolvable System: from biology to hardware, Prague (2008)
Onori M., Maffei A., Barata J., Hanisch C., Maraldo T.; "EUPASS- Adaptive Assembly Roadmap 2015"; Doc.D1.5f, EUPASS-Evolvable Ultra Precision Assembly, NMP-2-CT-2004-507978; May 2008

Onori M., Barata J.;"Evolvable Production Systems: Mechatronic Production Equipment With Process-Based Distributed Control"; Syroco 2009

Rosselli F.; "The Future of Manufacturing in Europe 2015-2020-The Challenge for Sustainability"; FutMan, Institute for Prospective Technological Studies, European Commission Joint Research Centre, EUR 20705 EN

Sandin E., Onori M.; "Enabling a process-oriented product design using an Assembly Module Platform"; Proceedings of the Integrated Design and Process Technology, IDPT-2002, Pasadena, USA, June 2002

"Strategic Research Agenda-assuring the future of manufacturing in Europe"; Manufuture PlatformExecutive Summary, Dec. 2005, EC

Wagner, G.P./Altenberg, L. 1996. Complex Adaptations and the Evolution of Evolvability Evolution 50(3): 967-976

WCED: Report of the World Commission on Environment and Development: "Our Common Future" Development and International Economic Co-Operation: Environment. United Nations (1987) 